\title{
3 Balancierung des Bewusstseins in der Lebensmitte
}

\author{
Dorothea Galuska
}

\section{Die Fragen der Lebensmitte}

Welche Fragen bewegen uns Menschen in der Lebensmitte, in einer Phase nach den Stürmen von Pubertät, nach unseren Ausbildungen, nach so manchen Beziehungstests und vor den Veränderungen des Alterns, des nicht mehr Arbeiten Müssens - sozusagen in einer oft als kreativ starken und unserer optimalen Schaffenskraft zugeschriebenen Lebenszeit? Vieles scheint durchlebt, ausgestanden oder errungen, manches festgezerrt und sicher, manches möglich und offen, anderes auch nicht mehr möglich oder nur unter großer Anstrengung - und: Das Ende wirkt absehbar, nicht mehr abstrakt, nicht als Gedankenspiel, sondern fühlbar, erkennbar; ich würde sagen: „sich zellulär anpirschend“.

- Was bedeutet diese Fühlung für unser Leben, für unser Bewusstsein?

- Wer bin ich wirklich - wirklich?

- Nicht nur im gestalteten Außen, sondern in meiner Innenwelt?

- Wo stehe ich im Leben, in meiner Arbeit, in der Kette der Generationen, im sozialen Gefüge meiner Kultur?

- Was ist das Leben überhaupt?

- Woher kommt es, wohin geht es? Und wieweit reicht mein Einfluss?

In meiner Begleitung von Menschen als Therapeutin, Führungskraft, als Unternehmerin, als Freundin, Partnerin und v.a. in meinem eigenen Leben stoße ich immer wieder neu auf diese Fragen und finde spiralartig immer wieder neue Antworten zu gleichen Fragekomplexen. Das ist faszinierend, denn auch wenn manche tiefen Werte ihren Wert für mich behalten, so wandeln sich doch die daraus abgeleiteten Lebens-Cestaltungen. 
Wenn wir uns nicht bereits freiwillig und neugierig in jungen Jahren oder durch schicksalhafte Herausforderungen der Blick- und Fühlrichtung nach innen zu wenden, dann oft in der Lebensmitte; manchmal mit der Begleiterscheinung einer sogenannten Midlife-Crisis, der Krise der Lebensmitte. Sie scheint - ähnlich wie die „dunkle Nacht der Seele“ für spirituell Suchende zumindest in milder Form unausweichlich, vergleichbar mit den Wachstums- und Wandlungsschmerzen der Pubertät.

Wenn ich beginne

- meine gewohnten Identifizierungen zu hinterfragen,

- meine Werte erneut zu überprüfen,

- die ganze Dimension des auf diesem Kongress schon oft zitierten Weltinnenraums zu erahnen,

dann schwanke ich zwischen Neugier, Ehrfurcht, Angst und Abwehr.

Und hier eine schlechte Nachricht:

\section{„Widerstand ist zwecklos"!}

\section{Umgang mit dem „Weltinnenraum“}

Wir können versuchen, Widerstand zu leisten, uns bemühen den inneren Rückruf zu überhören. Wir können mehr Kraft in äußere Inszenierungen legen, uns festhalten am Gewohnten, an unserer Leistungsorientierung, unserem Streben nach Wohlstand, Anerkennung oder Geld. Wir können uns auch betäuben, uns wegtrinken, eine Rundung mehr zulegen, oberflächlichem Spaß frönen - aber etwas in uns wird beginnen zu zweifeln, uns nicht mehr tragen. Die innere Stimme wird nicht leiser, wenn wir lauter werden. Aber sie ist gütig und sie wartet, denn sie weiß: „Widerstand ist zwecklos“.

Diese innere Instanz bedient sich unserer Träume, sie nutzt Momente unserer Muße und Kreativität, sie spricht zu uns im Ausdruck unserer Leidenschaft oder sie konfrontiert uns mit Gefühlen von Schalheit und Leere. Sie ist oft sehr präzise als Spiegel unserer Unstimmigkeiten, und sie beginnt oft schon lange vor dramatischen Krisen, sich auf subtile Weise in unserem Bewusstsein mitzuteilen. Bleiben wir im Widerstand, halten wir uns zu sehr fest am Altvertrauten, dann riskieren wir im immer größer werdenden Spagat zwischen äußerer und innerer Welt einen enormen Energieverlust, der uns auch in körperliche und seelische Krankheit führen kann, die uns dann umso deutlicher und gröber zur Einkehr oder Umkehr rufen wird.

Der dem äußeren Leben verhaftete identifizierte, vielleicht etwas zu sehr darin verlorene Teil oder sein Pendant, die widerständig-abwehrende Zurückweisung unserer Seele, wird in dieser Lebensphase wie zurückgerufen. So wie unsere Sinne, unsere Lust, Macht, potenzieller Reichtum, Image und andere Verführungen unseres Lebens uns nach Außen gerufen haben, so erfahren diese Qualitäten einen Rückruf aus der Tiefe unseres eigenen Inneren. Es geht dabei nicht um Polarisierung oder Abkehr, sondern um Balancierung und um Rückbindung.

Das was wir in Therapie und Spiritualität so gern als zu überwindende „Ego-Strukturen“ behandeln, könnten wir auch als ganz persönlich ausgelebte Weise der Intelligenz des Lebens betrachten und doch kann es sein, dass unsere alltäglichen Leiden- 
schaften und Freuden uns jetzt oft oberflächlich erscheinen, kurzlebig oder auch nicht wirklich erfüllend. Etwas fehlt. Und genau diese Sehnsucht ist der wunderbare Ruf der Seele in ihre eigene Tiefe. Sie ruft uns zu einer neuen Stimmigkeit und Authentizität. Nur - und das ist ein entscheidender Unterschied - müssen wir die Blick- und Fühlrichtung ändern, uns nicht mehr auf das Objekt unserer Sehnsucht ausrichten, sondern auf die Quelle, den Ursprung der Sehnsucht selbst.

\section{Das „Jenseitige“, Unbekannte und Ersehnte, von dem wir glaubten, es läge ir- gendwo „da draußen“" liegt bereits mitten in uns!}

\section{Spirituelle Inspirationen und Grenzen}

Im Buddhismus beginnen viele Lehrreden mit „Abgeschieden von den Sinnendingen ...", die meisten spirituellen Wege betonten Askese, ein Rückzug vom Weltlichen und von Leidenschaftslosigkeit. Gleichzeitig enthüllt und bewirkt aber gerade diese innere Abenteuer-Reise in die Tiefen unseres eigenen Bewusstseins eine völlig neue Dimension von Leidenschaft und Sinnestiefe. Die Aufmerksamkeits- oder Achtsamkeitslenkung nach Innen kann unsere inneren Schätze enthüllen. Sie „entschleiert“wie manche Mystiker es beschreiben und lässt uns Qualitäten wie Freude, Stille, Frieden, Offenheit oder Liebe in ihrer Reinheit fühlen. In der Tiefe unserer Seele, im Grund unseres Selbst liegen diese Erlebnisweisen als Potenzial und warten auf uns, sie zu vergegenwärtigen.

Wenn wir die Quelle mit der Oberfläche rückbinden, wenn wir uns selbst wiederfinden im liebenden Bezug zu dem was wir tun, dann finden wir auch unsere Erfüllung und unsere Leidenschaft wieder neu. Sie bezieht sich dann weniger auf das „was wir tun“ sondern auf das „wie wir es tun, aus welcher Haltung heraus“. Der Glanz des achtsamen, liebevoll zugewandten Tuns kann unsere Alltäglichkeiten zu Einzigartigem verwandeln.

Christliche Mystikerinnen, wie Mechthild von Magdeburg oder der begnadete Dichter Mevlana Rumi schrieben viele Zeugnisse dieser heftigen, oft auch körperlich empfundenen neuen Leidenschaft, sie sprechen vom göttlichen Geliebten oder dem süßen Liebhaber. Sie erlaubten ihrer Seele ganz in dieser großen Liebe aufzugehen.

Es geht also nicht um das Loswerden, das Ausschließen oder gar Vernichten von bisher Gelebtem, sondern um eine Umsortierung und Neuausrichtung, eine Vertiefung, einen Dimensionssprung.

\section{Wir werden das Leben mit seinen Höhen und Tiefen nicht los! Wir können es} anders betrachten und anders in ihm leben.

Schauen wir noch einmal zurück in unsere Leben:

Wie viele von uns besitzen noch ein mehr oder weniger durchgeliebtes Kuscheltier, das deutlich Spuren unserer einsamen Nächte, unserer Ängste und des gespendeten Trostes aufzeigen? Gerade diese Spuren von Intimität und Leidenschaft machen ihren besonderen Wert aus. Und dennoch hängen wir als Erwachsene nicht mehr so unerbittlich an der fühlbaren Form dieses Relikts unserer Kinderzeit. Was ist also passiert? 
Wir lernten, uns zu beobachten, zu bezeugen, die Perspektive zu wechseln. Wir lernten, dass es Dinge gibt, die anders und deswegen nicht „falsch“ sind. Wir lernten, wie wir und das Leben sich verändern. Wir lernten Zeit als zeitliche Abfolge - als Chronos - kennen. Wir lernten unser unmittelbares Fühlen in Denkmustern und Konzepte zu übersetzen.

\section{Das Potenzial der Lebensmitte}

Was könnten wir jetzt in der Lebensmitte lernen?

- Wir könnten einen individuellen spirituellen Weg beschreiten und Meditation als Bewusstseinstechnik ausprobieren.

- Wir könnten Bewusstseins-Räume ausloten wie uns unbekannte Länder.

- Wir könnten unseren Widerständen liebevoll begegnen und diese liebevolle Haltung selbst erkunden.

- Wir könnten die inneren Heilquellen, unsere inneren emotionalen Tankstellen finden und damit Atem- und Event-Pausen in unseren bewegten Leben erleben.

- Wir könnten lernen, unsere schnellen Gedanken zu beruhigen und Stille erleben.

Wer viel Zeit und Energie in den Erkenntnisweg der vertieften Meditation investiert, kann offene Weite und Leere in Bewusstseinsräumen fühlen. Diese tiefen Erfahrungen verändern unser Selbst- und Weltbild und sie bergen leider auch Gefahren von Selbstbezogenheit und Selbstüberschätzung, weil unsere Bindungsfähigkeit zu Menschen und unsere Liebesfähigkeit zur Welt auf dem Meditationskissen allein nicht wachsen werden.

Wer diese individuellen stillen Wege nicht mag, sucht sich vielleicht andere Herausforderungen:

den Jakobsweg gehen, Wüstenretreats, Extremsportarten, Selbsterfahrungsgruppen, Experimentieren mit psychoaktiven Substanzen, längere Auszeiten ohne die gewohnte Arbeit

- all das können Tore sein, um mit unserer Seele und der ihr innewohnenden Kraft und Wahrhaftigkeit in Kontakt zu kommen. Für manche von uns entsteht ein dann wieder hinderlicher Druck, fast Zwang zur Authentizität. Dann erliegen wir der Verabsolutierung eines an sich weich gemeinten Wertes. Oft ist die Lebensmitte eine Zeit der Neuausrichtung: Wir beenden Beziehungen, wenn wir uns ehrlich eingestehen, dass wir diesen Partner nicht oder nicht mehr wirklich lieben, wir suchen uns eine neue Arbeit oder Lebensausrichtung, die sich näher an der Wahrhaftigkeit orientiert.

Co-Präsenz, die Vergegenwärtigung unserer gemeinsamen Anwesenheit in all ihrer Unterschiedlichkeit und all ihrem Potenzial kann zum Schlüssel werden für sich verändernde oder langjährige Freundschaften und Beziehungen. Zum Verändern und Schmelzen unserer Beziehungsidentitäten und -muster brauchen wir die gegenseitige Ehrlichkeit und Bereitschaft, uns wirklich tief berühren und manchmal auch verletzen zu lassen. Die „blinden Flecken“ unserer Seele, unsere narzisstische Tendenzen entziehen sich zwar oft der eigenen Betrachtung, sind unserer Umgebung dafür umso spürbarer. Eine reife Seele scheut nicht den Freundschaftsdienst der Konfrontation - auch auf die Gefahr der Zurückweisung und im Wissen, dass die Tür 
zur Transzendenz nur von innen geöffnet werden kann. Und doch: Wenn ich mich selbst an den Platz meiner tiefen Offenheit bewege, kann ich zur Einladung werden für andere, ihre eigene Tür zu öffnen.

Es gibt zurzeit einige Gruppen, die bewusst mit solchen Co-Präsenz-Übungen und Forschungsweisen experimentieren, wie z.B. die von Almaas inspierierte Ridhwan School in Form der „dialectical inquiry“, einer Dialogform, die in das gemeinsame Feld der Beziehung forscht.

Hier geht es nicht mehr um „sich mögen“ oder nicht, um die Regulierung von Nähe und Distanz oder das Aushandeln von Ansichten, sondern um die Vergegenwärtigung dessen, was gerade da ist, so gut es beiden Dialogpartnern möglich ist. Beide stellen sich mitten hinein ins Leben.

Auch hierfür gibt es bereits historische Beispiele inniger spiritueller Freundschaften wie die von Rumi und Shams-e Tabrizi. Und so erfüllend und wundervoll diese sein mögen, es liegt auch in ihnen die Gefahr von Überhöhung und Ausschließlichkeit, die, wenn durchaus nicht beabsichtigt, so doch in ihrer Wirkung Zurückweisung und Eifersucht auslösen können. Man vermutet, dass Rumis hochverehrter Freund von Rumis eifersüchtigen Anhängern vertrieben, wenn nicht gar ermordet wurde.

\section{Offenheit für Tiefe, Licht und Schatten}

- Wie können wir Menschen also lernen, Tiefe zuzulassen, uns füreinander zu öffnen und gleichzeitig unsere Schatten erkennen?

- Wie können wir lernen von einer äußerlichen Moral zu einer in der Seele verankerten neuen Ethik zu gelangen, die uns selbst und unserer Umgebung Freiheit schenkt?

Das ist das Ringen der Lebensmitte. Es geschieht individuell und in unseren Beziehungen. Oft nicht gleichzeitig und auf unterschiedliche Weise in den Partnern einer Beziehung. Womit ein Partner ringt, kann für den anderen bereits gelöst sein oder noch kein Konfrontationsthema sein. Und dennoch ist es möglich, auch inmitten dieses Ringens eine eigene Schönheit und Ästhetik zu finden, wenn wir uns nicht ins bekannte Terrain von Widerstand und Weghaben-Wollen begeben.

\section{Ringen, tiefer verstehen wollen, Wachstums- und Reifungsschmerzen gehören zur Lebensmitte - sie sind Ausdruck dieser zu sich genommenen Lebensphase. Und wir können niemandem dieses Ringen abnehmen.}

Wir Therapeuten und spirituellen Gutmenschen fühlen uns oft vom Mitgefühl unserer Seele her verpflichtet $z u$ helfen, zu heilen, mitzutragen und laufen Gefahr, uns darin zu verlieren.

Manchmal entlastet das Situationen, aber wirkliche Veränderung geschieht erst, wenn ein Thema von innen her heilt, wenn die Grenze des Widerstands schmilzt, von innen aufgeweicht wird, eine Hingabe an die Weisheit des Lebens selbst erlebt wird. Dafür ist Co-Präsenz ausreichend und eigentlich die bessere Bedingung. Wenn 
ich offen bin für das Ringen und die Abgründe meines Partners, braucht auch er oder sie sich nicht zu bewerten, zurückzuweisen oder zu heilen.

\section{Das Leben selbst, die in ihr liegende Intelligenz findet ihren Weg.}

Und das ist dann auch die gute Nachricht.

„Widerstand ist wirklich zwecklos“. Die Liebe findet ihre Momente. Wir ringen mit der Unvollkommenheit, der Leidhaftigkeit, dem Schmerz des Lebens - und auch das ist Ausdruck des Lebens. Ausdruck der Vollkommenheit, die wir (noch) nicht als solche fühlen. Hier geschieht der Quatensprung in der Betrachtung.

\section{Eine neue Dimension erkunden}

Wenn wir aufhören, mit dem Leben und uns selbst in Reibung zu gehen, können wir Momente erleben, die sich trotz äußerer Unvollkommenheit vollkommen anfühlen. Wir können Augenblicke auch im tiefen Schmerz als „heil“ und ganz wahrnehmen. Die Durchlässigkeit und der Wechsel der Perspektiven ermöglicht uns eine umfassendere, weniger polarisierte oder einseitige Weise, das Leben zu uns zu nehmen. Sie erlaubt uns, einzigartig zu sein ohne uns zu Verabsolutieren, im Wissen und in Verbundenheit mit den Anderen und dem uns überschreitenden Unbekannten.

Auch unsere Arbeit und unsere Beziehungen erhalten durch diese Lockerung unserer Widerstände mehr Freiheit und eine neue innere Verankerung oder einen tieferen Sinn. Vielleicht spüren wir in uns selbst mehr Weichheit und Hingabe. Wer sich im seinem Handeln auf die Leere und das Unbekannte beziehen kann, erfährt aus dieser Öffnung manchmal sehr kreative Ideen, die nicht den gewohnten Bahnen und Mustern der bisher gestalteten Welt folgen.

Und schließlich kann uns diese Hingabe an das Leben eine Seinsweise eröffnen, die zu beschreiben sich aller Begrifflichkeit entzieht - auch wenn wir es immer wieder annähernd versuchen. Verlöschen, Erwachen, Erleuchtung, Ankommen, nach Hause kommen sind solche richtungsweisenden Begriffe für eine Bewegung an einen allumfassenden Ort.

\section{Transzendenz heißt für mich aber nicht nur Aufstieg und Überschreitung jeder Form ins Unbekannte, sondern auch eine gleichzeitige Rückbindung vom Nicht- wissen in eine lebbare Form.}

Als gesamte Menschheit befinden wir uns derzeit mitten in diesem Ringen, in einer Aufwach- und Neugestaltungsbewegung. Unsere Schatten und Widerstände begegnen uns überdeutlich, überall auf dem Erdball. Und wir wissen, dass es auch ums Überleben geht. Das globale Bewusstsein weiß um die Vernichtbarkeit nicht nur einzelnen Lebens, sondern unserer ganzen Art und unseres Planeten. Diese letztlich unvermeidbare Konfrontation zwingt uns dazu weiter zu experimentieren, andere Wege als die bisher bekannten zu entwickeln, co-kreativ zu werden, das Unbekannte $\mathrm{zu}$ versuchen, es gemeinsam zu suchen und vielleicht auch zu finden. 
Wahrscheinlich ist es schon da, als Potenzial - nur wir sind nicht da. Wir sind woanders verstrickt und verwickelt. Wir brauchen uns nicht noch mehr anstrengen; nur umfassender öffnen, uns wirklich aushalten und weiten. Freiheit ist immer frei- jederzeit.

Wie würde sich ein offenes globales Bewusstsein anfühlen und wie würde es Innen und Außen in eine liebevolle Verbindung bringen?

Wir könnten also die Lebensmitte zur Mitte unserer Innen- und Außen-Forschung werden lassen. Wir könnten unsere eigene Bewusstheit, unsere Co-Präsenz, unsere Welt-Präsenz in immer neue Formen des Miteinanders gießen.

Die Lebensmitte ist dann genau genommen keine zeitliche Phase im Sinne des „Chronos“, sondern eine immerwährende Dynamik des jeweils gelebten Moments als „Kairos“.

So verstanden ist die Lebensmitte der Ort, der Moment, an dem wir gerade sind. Jetzt - unabhängig von unserem realen Alter.

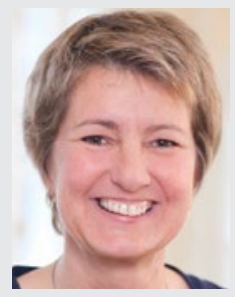

\section{Dorothea Galuska}

Leiterin des Personalmanagements der Heiligenfeld Kliniken, langjährige therapeutische Leiterin der Heiligenfeld Kliniken, Physio-, Bewegungs- und Körperpsychotherapeutin mit Ausbildungen in integrativer Bewegungstherapie und Orgodynamik, Europäisches Zertifikat für Psychotherapie (ECP), Meditationslehrerin, Schülerin der Ridhwan School (Almaas) 